\title{
Examination Method
}

National Cancer Institute

\section{Source}

National Cancer Institute. Examination Method. NCI Thesaurus. Code C81266.

A description of the technique for the inspection of a subject or object. 